\title{
Total Mixed Ration (TMR) Sampling Protocol
}

PETER H. ROBINSON, Dairy Cattle Nutritionist, Department of Animal Science, University of California, Davis; DEANNE MEYER, Livestock Waste Management Specialist, Department of Animal Science, University of California, Davis

This publication outlines a total mixed ration (TMR) sampling protocol that is practical for use on commercial dairy farms. Data from TMR samples may be used for several purposes, including to determine whether concentrations of nutrients meet identified standards.

The use of total mixed rations, rare until the early 1980s, is now the dominant feeding system on larger dairies in North America. TMR feeding has numerous advantages, including the ability to use lower-cost by-product feedstuffs at low levels in diets, completely mechanizing the feed mixing and delivery system and minimizing diet sorting by the cows.

However, if the TMR is not correctly mixed and fed, animal performance may be reduced by undersupplying critical nutrients, and environmental impacts may be increased (or money may be wasted) by oversupplying nutrients. There are times, under site-specific conditions, when it is valuable to sample TMR in order to compare results of the concentration of nutrients in feed delivered to the animals to a set of standard concentrations (i.e., formulated values). It is essential to establish a desired objective for use of TMR data before expending resources to collect samples. For some objectives, a different set of collected data may provide more accurate information.

\section{The Goal}

The goal of successful TMR sampling is to collect a representative sample of the TMR that was delivered to the feed bunk. It is necessary to collect handfuls of material from numerous locations to obtain a representative sample. Unlike sampling individual feed ingredients, which can be done at essentially any time of day, sampling of a TMR must occur within about 5 minutes of the unloading of the TMR at the feed bunk to ensure collection of a representative sample. Animal sorting of feed ingredients begins immediately after TMR delivery and can alter the nutrient content of the TMR. Therefore, the sample collector must be physically present at the time of TMR unloading to assure representative TMR sample collection. 


\section{Needed Equipment}

Equipment needed includes ( fig. 1)

- large plastic bucket

- pair of latex gloves

- plasterer's spatula

- 1-gallon size plastic bag with zip-type closure

- whisk broom

- black permanent marker

- notebook

- scissors

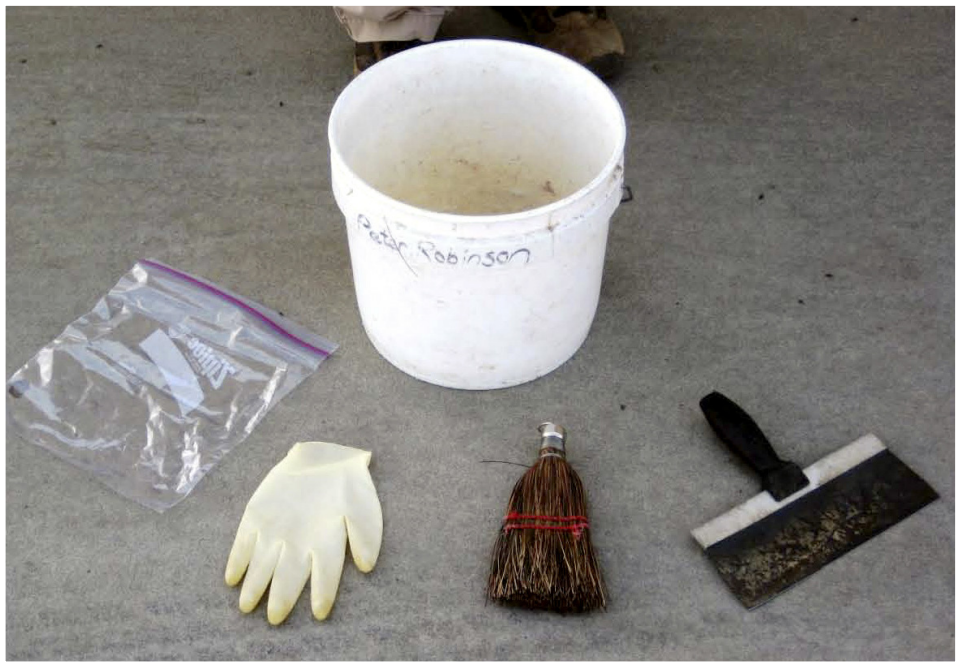

Figure 1. Equipment needed.

\section{Collect Increment Samples at the Bunkline}

Step 1. Identify the sample. Label the sample bags with a permanent marker to include sample identification, date and time of sampling, initials of the person taking the sample, the type of material sampled, and the analyses to be performed. In a notebook, record the bunk and ration identification, along with the sample ID to be used by the laboratory.

Step 2. Identify increment sampling sites. Ten individual samples are needed to create a composite sample. In each bunker to be sampled, imagine the appropriate number of sampling locations at regular intervals along the entire bunkline to collect ten samples. For example, collect samples at every tenth stanchion or every third support post.

Step 3. Collect the composite sample. Follow the feed truck at a set, safe distance with your plastic bucket and collect individual samples at the identified sites ( fig. 2). Insert a latex-gloved hand into the middle of the pile about wrist deep ( fig. 3). Grab and retrieve each increment sample. Collect ten per bunk. Do not squeeze or shake these before dropping them into the plastic bucket. When collection is complete, gently compress the material in the bucket and place a plastic bag on top to prevent moisture loss.

\section{Tips}

- Safety is always a concern. Be sure all appropriate individuals are notified that someone will be following the feed truck to sample the TMR. Individuals sampling TMR should directly identify themselves to the feed truck driver. Wearing bright colors may help.

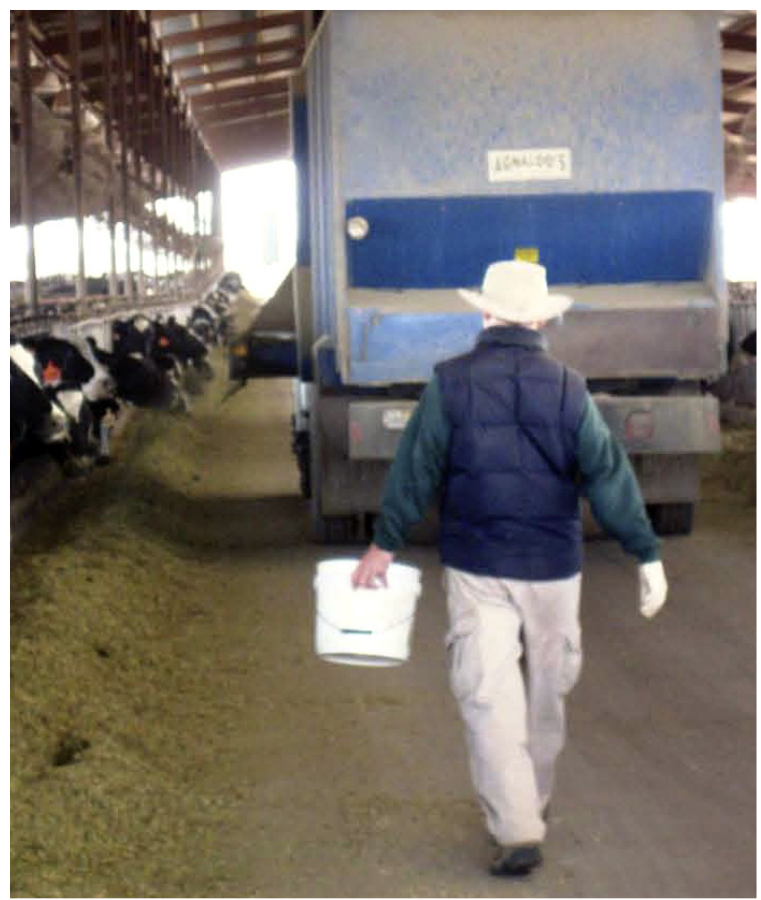

Figure 2. Following the feed truck. 


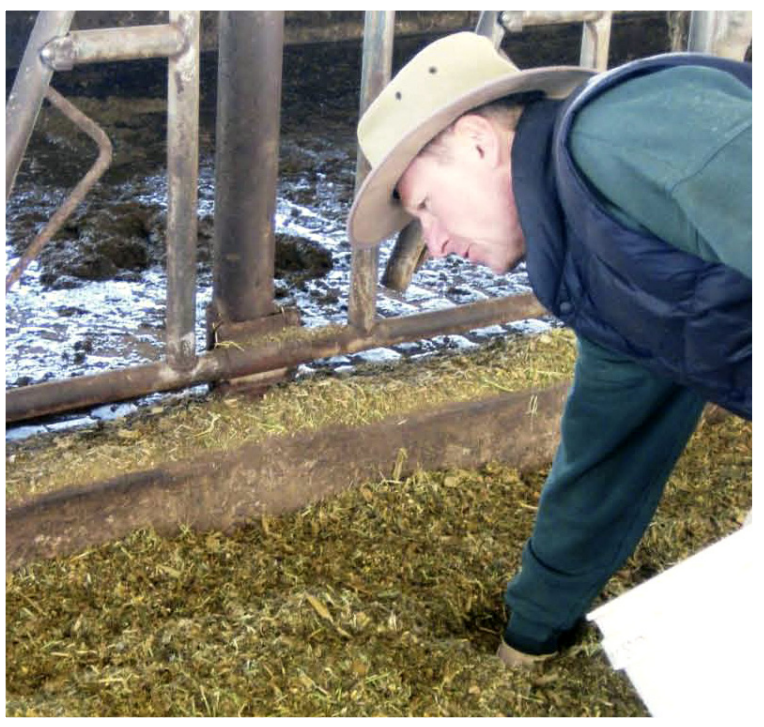

Figure 3. Sampling the bunkline.

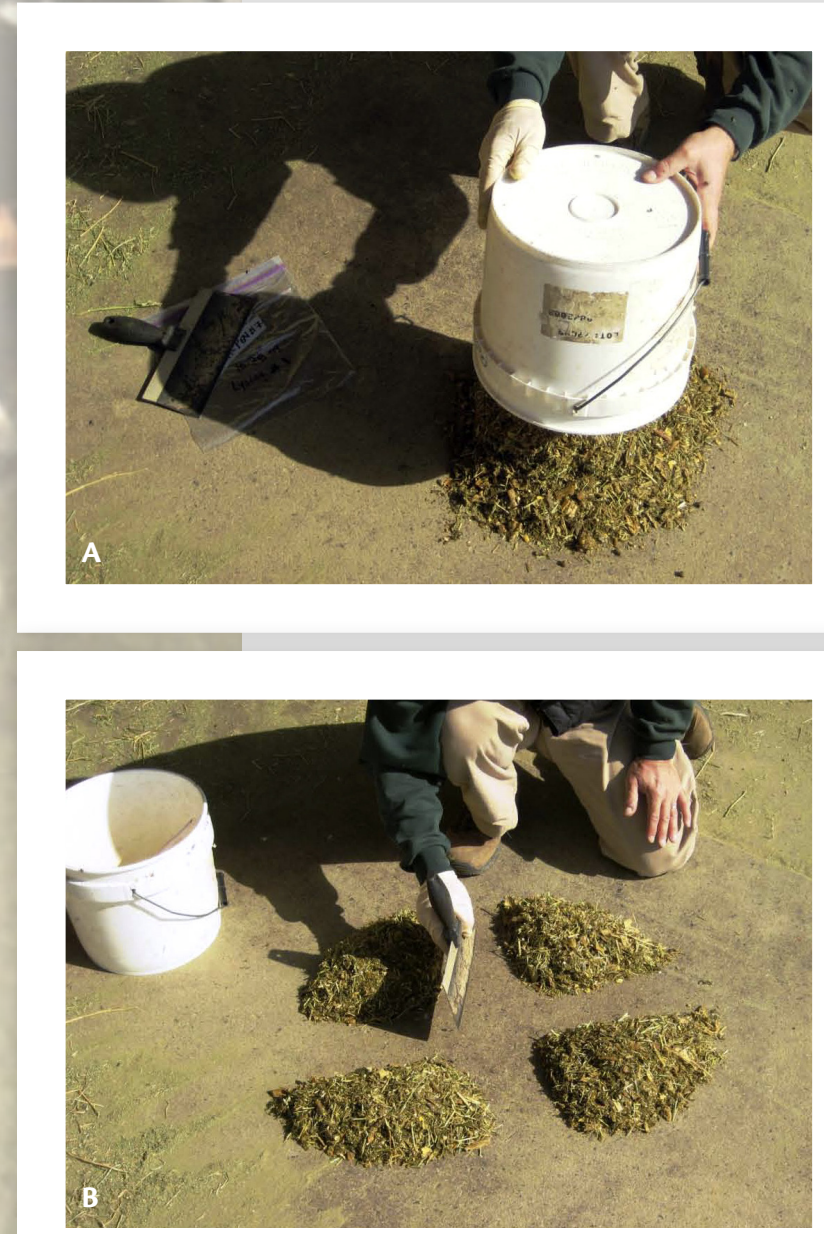

Figure 4. Dumping (A) and quartering (B) sample. The intermediate stage of mixing is not shown.
- Do not avoid oversized feedstuffs-large by-product feed ingredients such as whole carrots, citrus, etc.--during sampling.

- Clip long forages, such as straws or grass hays, that do not break up in mixing to about 1 inch from the mass prior to putting them into the bucket in order to avoid overrepresentation of these dietary components in the TMR sample.

\section{Prepare the Laboratory Sample}

The composite sample in the bucket is too large to submit for laboratory analysis. It is necessary to mix, quarter, bag, and preserve a representative subsample.

Step 4. Mix the composite sample. Sweep a few square feet of a smooth concrete or composition surface, which is protected from wind and sun, clean with your whisk broom. Dump the contents of the bucket onto this clean area (fig. 4A). Mix the material thoroughly with the plasterer's spatula by repeatedly turning the TMR inward from the bottom to the top until it has a well-mixed homogenous appearance. It may be necessary to tear, slice, or clip large ingredients (such as citrus pulp, carrots, or long forages) with the plasterer's spatula or a pair of scissors into quarter-sized pieces or 1-inch lengths.

Step 5. Quarter the composite sample. Use the plasterer's spatula to separate the well-mixed pile into halves and then into quarters ( fig. 4B).

Step 6. Bag the laboratory sample. Place two diagonally opposing quarters into the gallon plastic bag, squeeze out excess air, and seal it tightly ( fig. 5). Bag the remaining feed as a reserve sample or return it to the feed bunk. Be sure to sweep up small particles with the whisk broom onto the spatula and include them in the laboratory samples. 

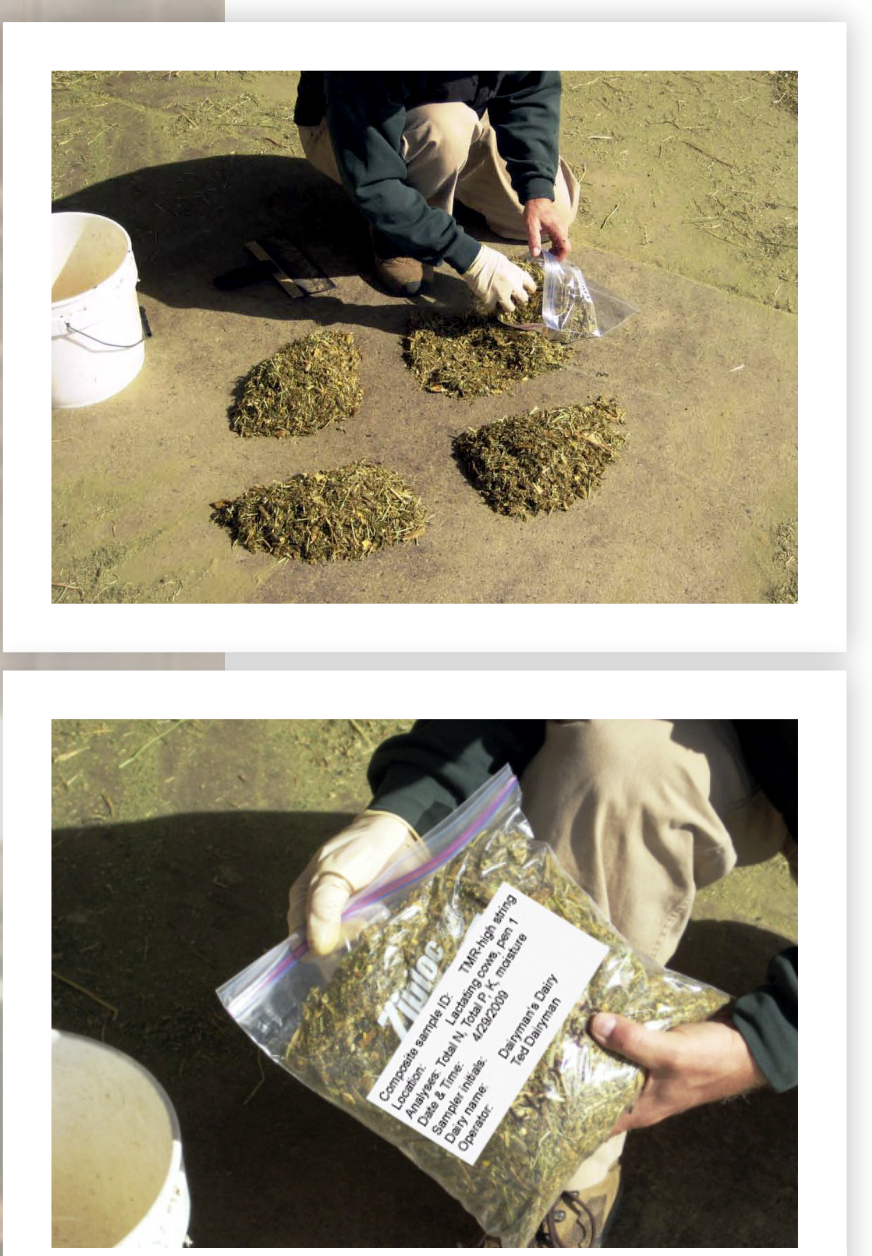

Figure 5. Creating the final sample. Fill bag with contents from opposing quarters.
Step 8. Submit the laboratory sample to the laboratory. Work with your dairy nutritionist to determine the appropriate analyses. Standard laboratory analysis packages may not provide the information needed for your objectives.

\section{Frequency of Sampling a TMR}

Work with your dairy nutritionist to determine the appropriate frequency at which to collect the TMR. Collect samples regularly to establish sufficient historic data to meet your desired objectives.

\section{Use of Results}

The value of TMR sample analysis, at least in a general sense, is to establish a pattern of the concentration of nutrients in the TMR delivered to a group of animals over time. Comparing the results of sampled individual feeds with standard or formulated values may be of value in select situations. Confidence in TMR formulation, and the mixing and delivery processes, will be high when the assayed TMR nutrient concentrations are similar to the formulated values. Additional systematic evaluation of the TMR feeding system may be warranted if analytical nutrient results of the TMR regularly vary from formulated values.

\section{Preserve and Submit the Laboratory Sample to an Analytical Laboratory}

Proper preservation and storage is critical to obtaining useful results. Feed ingredients may degrade rapidly under warm or moist conditions, making the analytical data inaccurate.

Step 7. Cool the laboratory samples. Place sealed samples in an ice-cooled chest or refrigerator and deliver them to the laboratory within 24 hours. Alternatively, these samples may be frozen if delivery to the laboratory requires more than 24 hours. 


\section{For Further Information}

To order or obtain ANR publications and other products, visit the ANR Communication Services online catalog at http://anrcatalog.ucdavis.edu or phone 1-800-994-8849. You can also place orders by mail or FAX, or request a printed catalog of our products from

University of California

Agriculture and Natural Resources

Communication Services

1301 S. 46th Street

Building 478 - MC 3580

Richmond, CA 94804-4600

Telephone 1-800-994-8849

510-665-2195

FAX 510-665-3427

E-mail:danrcs@ucdavis.edu

(C)2010 The Regents of the University of California

Agriculture and Natural Resources

All rights reserved.

No part of this publication may be reproduced, stored in a retrieval system, or transmitted, in any form or by any means, electronic, mechanical, photocopying, recording, or otherwise, without the written permission of the publisher and the authors.

Publication 8413

ISBN-13: 978-1-60107-703-5

The University of California prohibits discrimination or harassment of any person on the basis of race, color, national origin, religion, sex, gender identity, pregnancy (including childbirth, and medical conditions related to pregnancy or childbirth), physical or mental disability, medical condition (cancer-related or genetic characteristics), ancestry, marital status, age, sexual orientation, citizenship, or service in the uniformed services (as defined by the Uniformed Services Employment and Reemployment Rights Act of 1994: service in the uniformed services includes membership, application for membership, performance of service, application for service, or obligation for service in the uniformed services) in any of its programs or activities.

University policy also prohibits reprisal or retaliation against any person in any of its programs or activities for making a complaint of discrimination or sexual harassment or for using or participating in the investigation or resolution process of any such complaint. University policy is intended to be consistent with the provisions of applicable State and Federal laws.

Inquiries regarding the University's nondiscrimination policies may be directed to the Affirmative Action/Equal Opportunity Director, University of California, Agriculture and Natural Resources, 1111 Franklin Street, 6th Floor, Oakland, CA 94607, (510) 987-0096. For information about ordering this publication, telephone 1-800994-8849. For assistance in downloading this publication, telephone 530-754-3927.

An electronic copy of this publication can be found at the ANR Communication Services catalog Web site, http://anrcatalog. ucdavis.edu.

\section{UCC}

REVIEWED This publication has been anonymously peer reviewed for technical accuracy by University of California scientists and other qualified professionals. This review process was managed by the ANR Associate Editor for Animal, Avian and Veterinary Sciences.

web-8/10-SB/RW 